\title{
An Amplitude-normalized Pseudo Well-log Construction Method and its Application on AVO Inversion in a Well-absent Marine Area
}

\author{
Chunyan $\mathrm{FAN}^{1}$, Yan $\mathrm{SONG}^{1}$, Yuanyin $\mathrm{ZHANG}^{1,2}$, and Zhenxue JIANG ${ }^{1}$ \\ ${ }^{1}$ China University of Petroleum - Beijing, Beijing, China \\ ${ }^{2}$ Petroleum Exploration \& Production Research Institute, SINOPEC, Beijing, China \\ e-mail: yuanyinshou@163.com (corresponding author)
}

\section{Abstract}

AVO inversion is hard to be efficiently applied in unexploited fields due to the insufficiency of well information. For the sake of AVO inversion in a well-absent area, the most conventional method is to construct pseudo well-logs by defining seismic processing velocity as the $P$-velocity and computing $S$-velocity and density using empirical formulas, yet the resolution of the corresponding earth models and final inverted results could be extremely low, and a rough formula could destroy the inversion thoroughly. To overcome this problem, an amplitudenormalized pseudo well-log construction method that reconstructs pseudo well-logs in accordance with computed $P$-wave reflection amplitudes and nearby drilling data is proposed in this paper. It enhances the inversion resolution efficiently with respect to the real elastic parameter relationships, so that the corresponding AVO inversion results are reasonably improved. In summary, the proposed method is successfully applied in the AVO inversion of a well-absent marine area, and could be valuable in the early phase, particularly of the offshore hydrocarbon exploration.

Key words: well-absent pre-stack seismic AVO inversion, marine exploration, reservoir appraisal, pseudo well-logs reconstruction. 


\section{INTRODUCTION}

The pre-stack seismic AVO inversion is better for reservoir prediction than post-stack methods owing to its preservation of the AVO information and more elastic parameters (Connolly 1999, Zhang et al. 2011b), but it cannot be efficiently conducted if the well information is insufficient to accurately scale the wavelets and build constrained elastic models. Because the constrained models essentially represent initial predictions to construct inversion, and can lead to a better resolvability and link between the seismic data and the actual lithology (e.g., from logging data and geological mapping), the inversion result of predicted sediment properties is actually a set of elastic volumes that deviate as little as possible from the initial predictions, while at the same time they are modeling the real geology as closely as possible (Hampson et al. 2001, 2005).

For a convenient application of AVO inversion method in a well-absent area, the most conventional way is to construct some pseudo well-logs by defining interval velocities obtained from 3D seismic processing stage, initially as the $P$-velocities, and then compute $S$-velocities and densities using empirical formulas. However, by this method the resolutions of corresponding earth models and final results directly inverted from seismic processing velocities could be extremely low, and using a rough formula for elastic parameters prediction could further destroy the inversion (Zhang et al. 2011b, Fan et al. 2013).

In this paper, in order to increase the resolution of well-absent AVO inversion results and hence retrieve reliable elastic parameters for reservoir description, we propose a new pseudo well-log construction method and apply it in an undrilled marine study area, where quite promising but not wellstudied reef and turbidity sand reservoirs need to be appraised for further exploration.

\section{GEOLOGICAL SETTING AND DATA SETS}

As shown in Fig. 1, the study field is located in the northwestern segment of South China Sea. It lies offshore southern Hainan trending northwestsoutheast with an areal extent of around $200 \mathrm{~km}^{2}$; the nearest drilling site is about $60 \mathrm{~km}$ away from the study area, marked as well $\mathrm{Y}-1$ on the index map. The water depth gradually increases from $1.2 \mathrm{~s}$ (around $600 \mathrm{~m}$ ) in the northwest to $1.6 \mathrm{~s}$ (around $800 \mathrm{~m}$ ) in the southeast, whereas a seabed pit can be distinctly seen in the southern segment.

The pre- and post-stack 3D seismic data quality is quite good for seismic stratigraphic interpretation and AVO analysis. The bin size of CMP gathers is $12.5 \times 25 \mathrm{~m}$, with a full fold of 140 . As shown in Fig. 2, the seismic traces are characterized by a frequency content from 8 to $75 \mathrm{~Hz}$ with the predomi- 


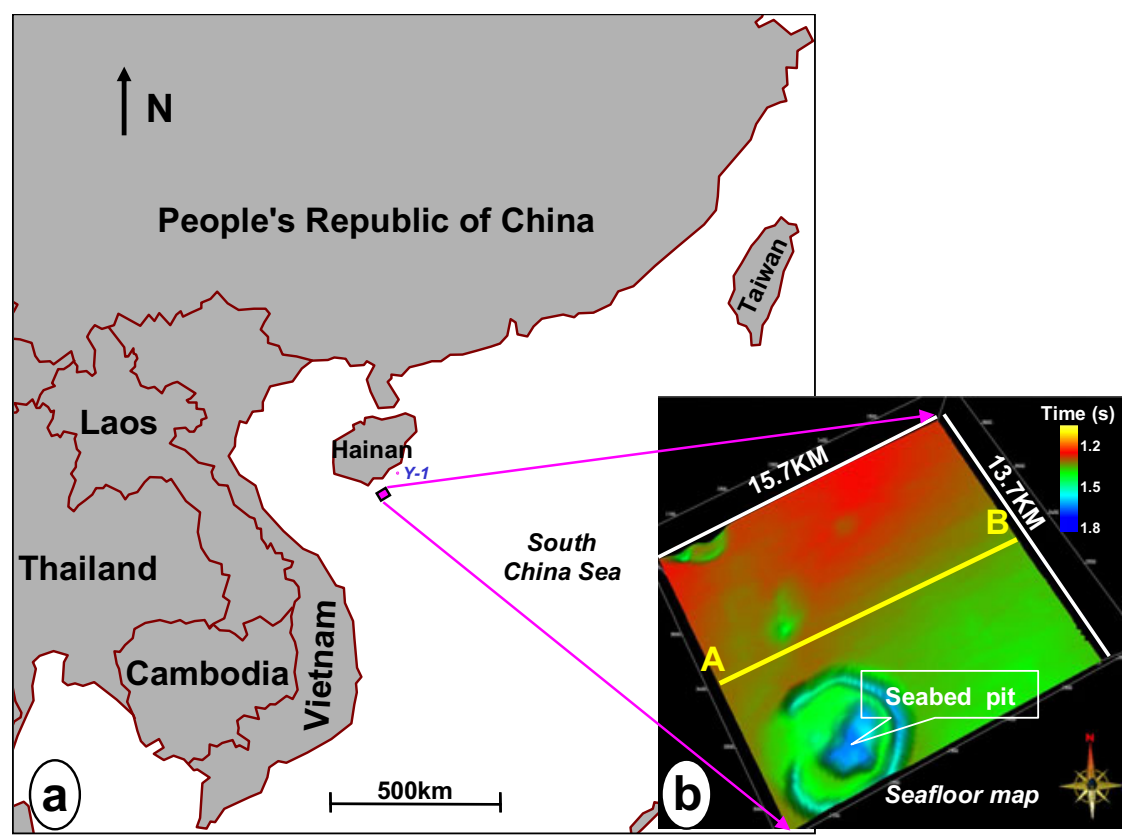

Fig. 1. Location of the study field, about $60 \mathrm{~km}$ away from Well Y-1 (a). Its seafloor map (b) shows that the water depth gradually increases from $1.2 \mathrm{~s}$ (around $600 \mathrm{~m}$ ) in the northwest to $1.6 \mathrm{~s}$ (around $800 \mathrm{~m}$ ) in the southeast, whereas an obvious seabed pit exists in the southern segment.

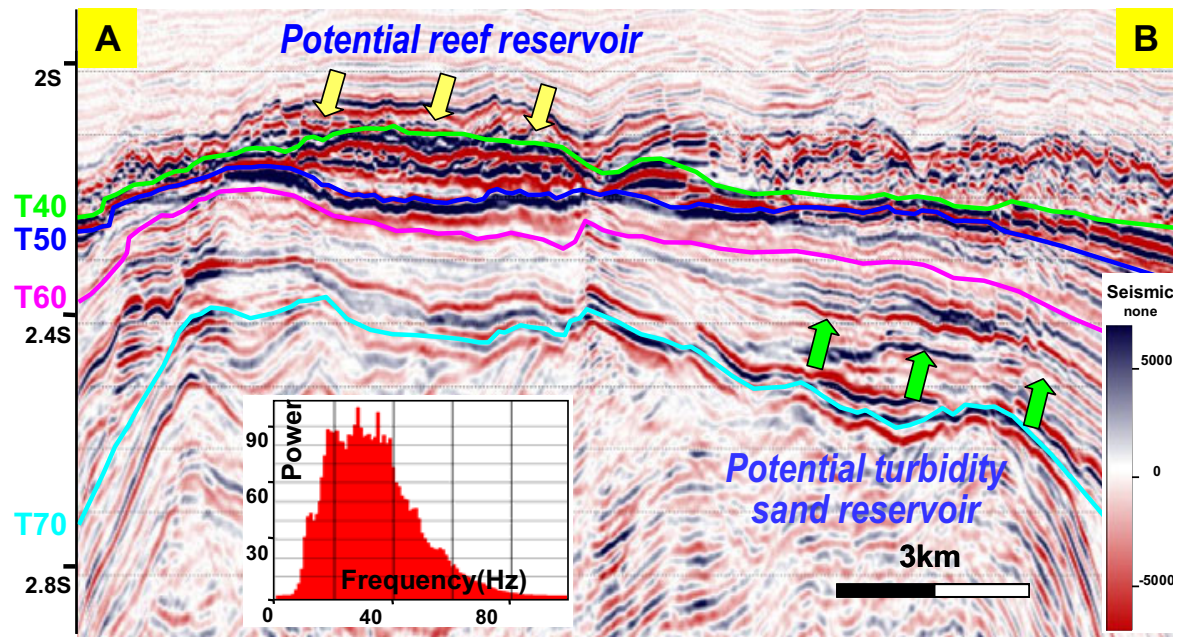

Fig. 2. The typical seismic section following line AB in Fig. 1b. Two sets of potential reservoirs, separately marked by yellow and green arrowheads, need to be finely described for further exploration. 
nant frequency of $34 \mathrm{~Hz}$. The main stratigraphy summarized from the nearest drilling area is listed in Table 1. It indicates that Meishan (T40 to T50) and Lingshui (T60 to T70) fm are the two target formations, prone to develop reservoirs in carbonate reef and turbidity sand units, respectively.

Table 1

Stratigraphy summarized from the nearest drilling location

\begin{tabular}{|c|l|l|c|}
\hline System & \multicolumn{1}{|c|}{ Series } & Formation & Horizontal \\
\hline \multirow{5}{*}{ Neogene } & Holocene & & \\
& Pleistocene & & T20 \\
& Pliocene & Yinggehai & T30 \\
\cline { 2 - 3 } & \multirow{3}{*}{ Miocene } & Huangliu & T40 \\
& & Meishan & T50 \\
& & Sanya & T70 \\
\cline { 2 - 3 } Oligocene & Oligocene & Lingshui & T80 \\
& Eocene & Yacheng & \\
& & Lingtou & \\
\hline
\end{tabular}

The top and base units of two potential reservoir intervals can be clearly identified from the post-stack seismic reflection data by their very strong reflection amplitudes (Fig. 2); the time thickness distributions of Meishan and Lingshui formations (Fig. 3) have roughly revealed that both of the two sets of reservoirs lie in an area of large extent. Unfortunately, AVO inversion cannot be efficiently applied to retrieve valuable elastic parameters for reser-

(a)

(b)
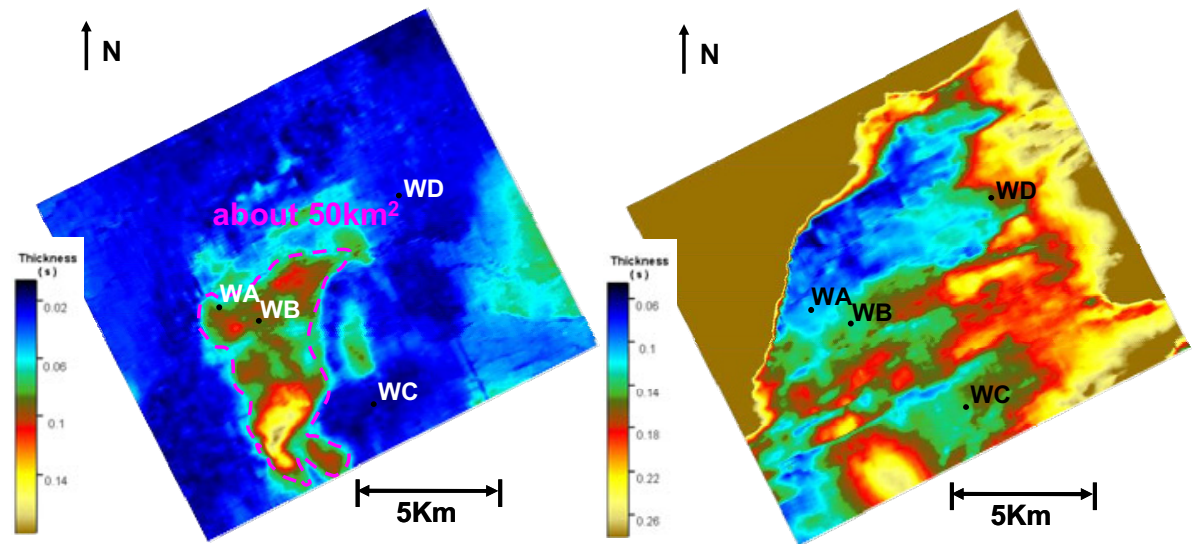

Fig. 3. The time thickness maps of Meishan (T40-T50) (a), and Lingshui (T60-T70) fm (b). WA, WB, WC, and WD are the four pseudo wells. 
voir description since there is no well currently drilled in the study area. The drilling cost and risks in a marine environment, especially in deep waters, are actually a few times higher than that of land exploration. Therefore, it is crucial not only to perform a detailed reservoir assessment or cautious welldeployment but also to carry on an AVO study without well-logging data. On the contrary, the lack of the logging data in the early exploration phase also restricts the reservoir prediction accuracy. This common contradiction is essentially the key problem in the study area.

\section{METHODS}

Different from the conventional method by directly defining seismic processing velocity as the $P$-velocity and computing $S$-velocity and density using empirical formulas, the new method proposed in this paper reconstructs, at first, the pseudo well-logs incorporated with computed $P$-wave reflection amplitudes and an actual well-log data from the nearest drilling site. It then conducts pre-stack simultaneous AVO/AVA inversion for a high-quality description of elastic reservoir parameters. The $P$-wave data are computed from CRP (common reflection point) gathers by AVO inversion. As shown in Fig. 4, this method consists of three major approaches, including the initial pseudo well-logs construction, reconstruction, and AVO inversion.

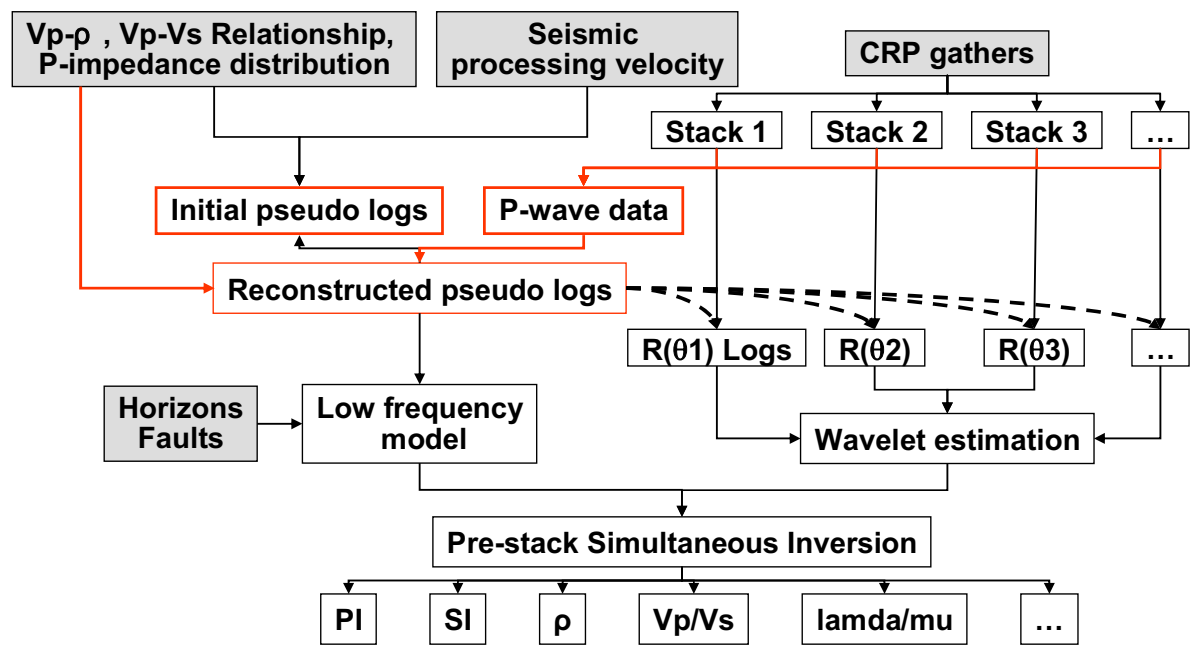

Fig. 4. The well-absent AVO inversion workflow based on amplitude-normalized pseudo well-logs construction method. The original data are marked by gray shades. The $V_{p}-\rho$ and $V_{p}-V_{s}$ relationships, as well as $P$-impedance distribution, are achieved from nearby drilling data. The $P$-wave data computed from CRP gathers by AVO inversion have a higher resolution, and are more beneficial for reservoir prediction and pseudo well-logs reconstruction. 


\subsection{Initial pseudo well-logs construction}

The first step, constructing $P$-velocity for a pseudo well from interval velocity, is probably the same as in the conventional method. Although the interval velocities defined in 3D seismic processing stage could be carefully and densely picked, its dominant frequency (related to seismic velocity picking, often less than $5 \mathrm{~Hz}$ ) is still inferior to that of seismic data (around 20-40 Hz), and far below that of logging data (often $>1000 \mathrm{~Hz}$ ). Thus, the corresponding final inverted results are liable to have low resolutions and incorrect predictions.

Besides, as mentioned above, using a rough formula for elastic parameters prediction could destroy inversion thoroughly (Zhang et al. 2011b, Fan et al. 2013). Therefore, comparing to empirical formulas (for instance, Castagna's mud-rock line and Greenberg-Castagna's $V_{p}-V_{s}$ relationship, etc.; Lamb et al. 1992), it is preferable to use the $V_{p}-V_{s}$ and $V_{p}-\rho$ relationships summarized from nearby drilling data to compute $S$-velocity and density, respectively.

\subsection{Pseudo well-logs reconstruction}

To reasonably improve the inversion resolution, the amplitude-preserved seismic data, drilling information and seismic processing velocity are integrated to reconstruct pseudo well-logs. The basic methodology is that the magnitudes and signs of seismic reflections are correlative with those of the subsurface layers if the stacked seismic data are amplitude-preserved. The reflectivity variations are also related to the changes of $P$-impedance $(P I)$. The pseudo $P I$ well-logs can therefore be reconstructed by converting fullstack seismic reflection to elastic parameters following the $P I$ tendency.

\subsubsection{P-wave data computation}

As the full-stacked data have made a reluctant compromise to data resolution sacrificing due to the contamination of AVO effects no matter how much the SNR degree could be enhanced in the stacking process (Zhang et al. 2013), we compute the $P$-wave data from CRP gathers by AVO inversion to make a corroborating substitution, based on Eq. 1 (Gidlow 1992, Sun 1999):

$$
R_{p p}(\theta)=\left(1+\tan ^{2} \theta\right) R_{p}-8 \frac{V_{s}^{2}}{V_{p}^{2}} R_{s} \sin ^{2} \theta-\left(\frac{1}{2} \tan ^{2} \theta-2 \frac{V_{s}^{2}}{V_{p}^{2}} \sin ^{2} \theta\right) \frac{\Delta \rho}{\rho},
$$

where $R_{p}$ and $R_{s}$ are theoretical zero-offset $P$-wave reflectivity and $S$-wave reflectivity, respectively,

$$
R_{p}=\frac{1}{2}\left(\frac{\Delta \rho}{\rho}+\frac{\Delta V_{p}}{V_{p}}\right), \quad R_{s}=\frac{1}{2}\left(\frac{\Delta \rho}{\rho}+\frac{\Delta V_{s}}{V_{s}}\right),
$$


and $V_{p}, V_{s}$, and $\rho$ are the average $P$-wave velocity, $S$-wave velocity, and density of the reflection boundary, respectively. $\theta$ is the average of incidence and transmission angles, $R_{p p}(\theta)$ is the elastic reflectivity with ray-path of incident angle, $\Delta \rho / \rho$ is the density gradient, and $V_{s} / V_{p}$ is the $S$-to- $P$-wave velocity ratio.

For solving the non-linear Eq. 1, the $P$-wave result is comparatively stable since the coefficient of $R_{p}$ is usually bigger than that of $R_{s}$ or $\Delta \rho / \rho$, and has nothing to do with $V_{p} / V_{s}$ (Zhang et al. 2013). If we use seismic amplitudes of CRP gathers to substitute $R_{p p}(\theta)$, then the computed corresponding $R_{p}$ results are the zero offset $P$-wave reflection data. Comparing to conventional full-stack data, the computed $P$-wave data have a higher resolution and are more beneficial for reservoir prediction (Zhang et al. 2013), inevitably for pseudo well-logs reconstruction.

\subsubsection{P-impedance tendency computation}

The $P$-impedance tendency $\left(P I_{0}\right)$ is computed by the multiplication between seismic interval velocity and density, while density is computed from the statistical relationship of the nearest drilling data instead of any empirical formulas.

\subsubsection{Pseudo well-logs reconstruction}

The amplitude-normalized pseudo-well log reconstruction equation for each sample in each trace can be listed as follows:

$$
\frac{x-0}{B-A}=\frac{P I-P I_{0}}{D-C},
$$

where $P I$ is the reconstructed $P$-impedance, $P I_{0}$ is the $P$-impedance tendency, and $x$ is the reflection amplitude for $P$-wave data in that sample. $[A, B]$ and $[C, D]$ are the distribution ranges of computed $P$-wave reflection amplitudes and recorded $P$-impedance values from the nearby drilling data, respectively. So,

$$
P I=\frac{1}{B-A}\left((D-C) x+(B-A) P I_{0}\right),
$$

The density and $S$-impedance of pseudo well-logs can be computed from the statistical relationships from nearby drill well logs too.

\subsection{AVO inversion conduction}

With these reconstructed pseudo well-logs, the pre-stack simultaneous AVO/AVA inversion can be conducted to achieve a great number of elastic parameters such as $P I, S I$ ( $S$-impedance), $V_{p} / V_{s}$, Poisson ratio, $\lambda \rho$, and $\mu \rho$, 
etc., for reservoir prediction. The reconstructed curves have higher dominant frequencies (same as that of seismic data), which could be definitely helpful for scaling wavelets and building initial models. The final AVO inverted results could therefore be improved.

\section{COMPARISON OF RESULTS}

Figure 5 shows the interval velocity profile defined in seismic processing stage of study area in the same line as in Fig. 2. It has been carefully and densely picked, with an interval of $100 \times 100 \mathrm{~m}$. Besides, the amplitude of CRP gathers analyzed in this study is well-preserved and qualified for $P$-wave data computation via AVO inversion because of the careful seismic data processing, including signal to noise ratio (SNR) enhancement, consistency and vertical resolution improvement, amplitude-preserved pre-stack migration, as well as data conditioning (Zhang et al. 2011a,b).

As shown in Fig. 6, comparing with conventional full-stack data (Fig. 6a), the inverted $P$-wave data from CRP gathers by AVO inversion (Fig. 6b) have a higher resolution with more clear and continuous events. The dominant frequency of inverted $P$-wave data is $39 \mathrm{~Hz}$, while the counterpart of stack data is only $34 \mathrm{~Hz}$ on the whole section. This enhancement is even higher in some target zones, and crucial for pseudo well-logs reconstruction.

Figure 7 shows the comparison of seismic (a), synthetic (b), constructed $P$-impedance $(P I)(\mathrm{c})$, and extracted wavelet (d) by conventional method for pseudo-well WC. The constructed pseudo PI log inevitably has a very low

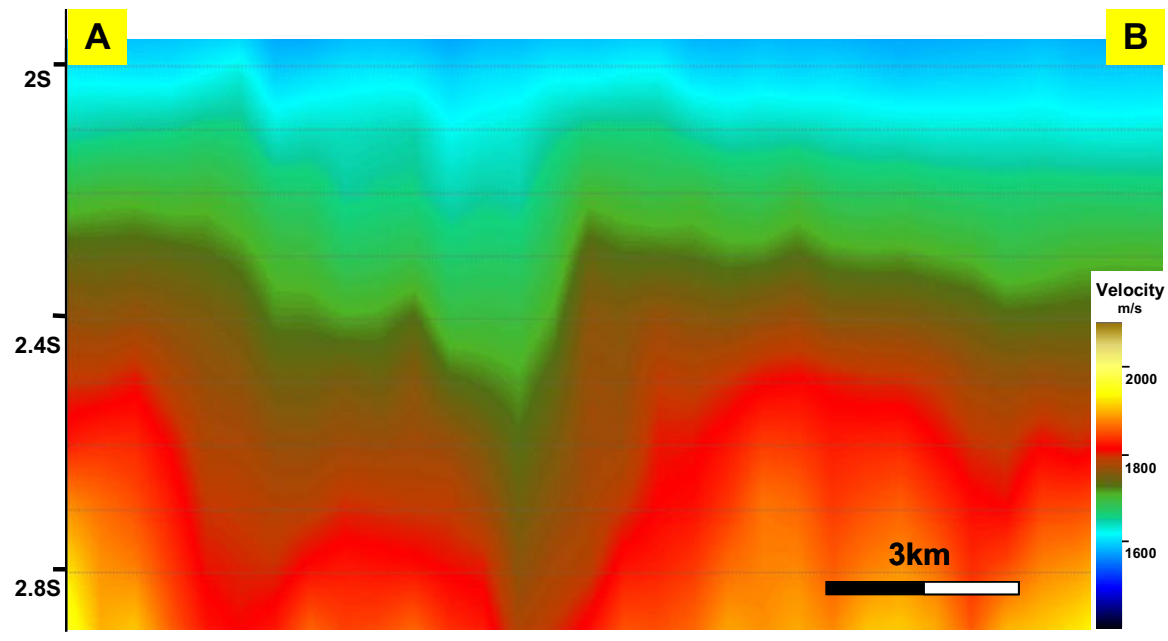

Fig. 5. The seismic processing velocity profile in the same line as in Fig. 2. 

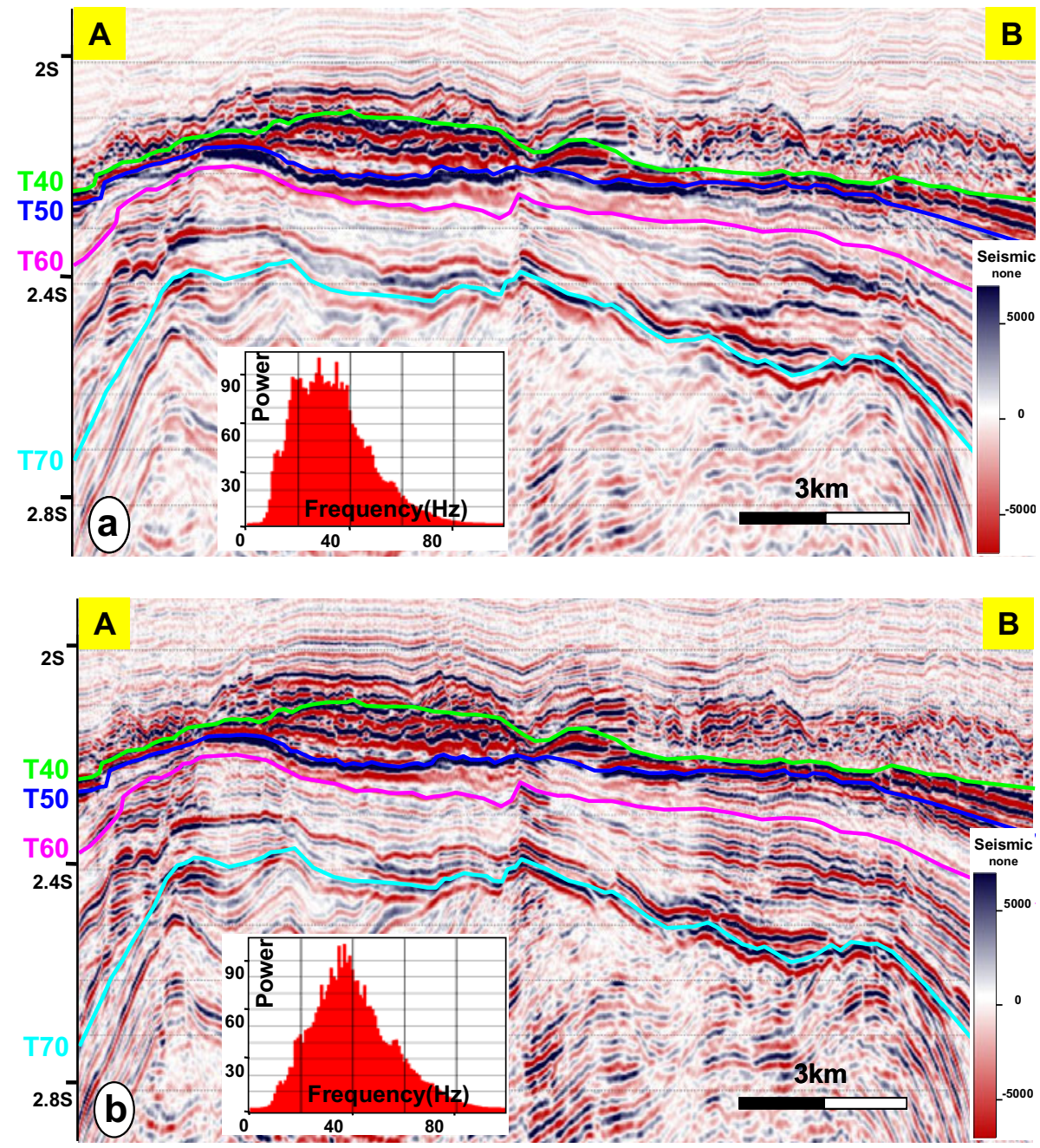

Fig. 6. Comparison between full-stack section (a) and inverted $P$-wave section (b) across the same profile as in Fig. 2. After the removal of AVO effects, seismic reflection events are more clear and continuous on the inverted $P$-wave section, and the dominant frequency is enhanced by $5 \mathrm{~Hz}$ compared with full-stack data across the whole section.

frequency bandwidth and definitely lost many details, thus the extracted wavelet energy has to be increased to compensate for the tiny reflection variations. Consequently, it is failed to produce a qualified correction between synthetic and seismic data. The extraction of reflection coefficients in 


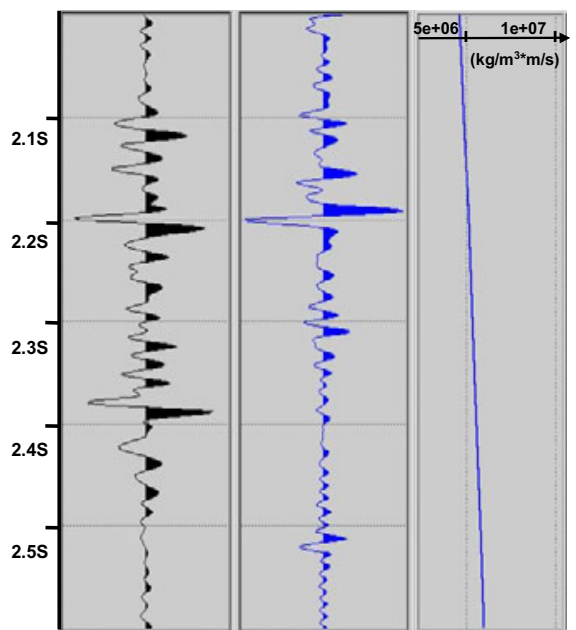

(a ) Seismic (b) Synthetic (c) P
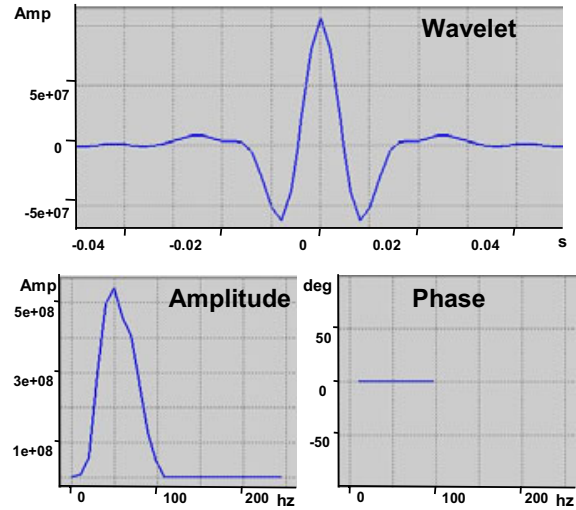

(d) Wavelet

Fig. 7. The comparison of seismic (a), synthetic (b), constructed pseudo $P$-impedance $(P I)(\mathrm{c})$, and extracted wavelet (d) by conventional method for pseudo-well WC. The constructed pseudo $P I$ well-log with an extremely low dominant frequency has inevitably smoothed many details and irrationally boosted up the energy of extracted wavelets, which subsequently cause a bad-correlation between synthetic and seismic data.

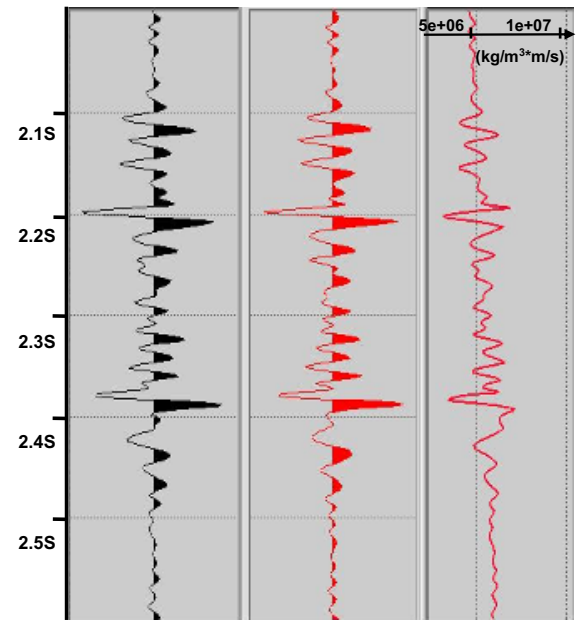

(a) Seismic (b) Synthetic (c) PI
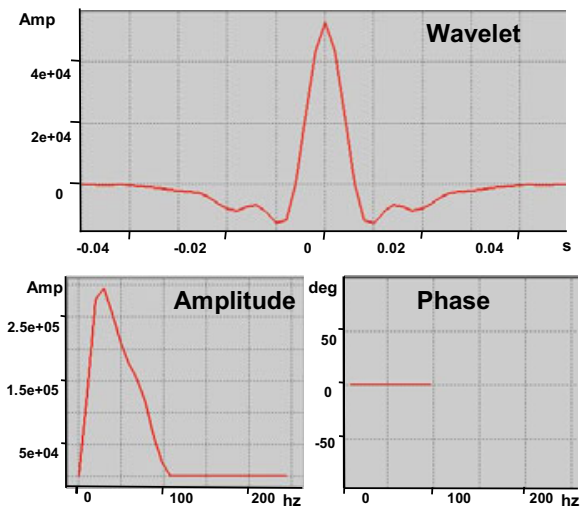

(d) Wavelet

Fig. 8. The comparison of seismic (a), synthetic (b), reconstructed pseudo $P$-impedance $(P I)(\mathrm{c})$, and extracted wavelet (d) by our proposed method for pseudo-well WC. The dominant frequency of pseudo well-log is higher than that of conventional counterpart (Fig. 7c). 

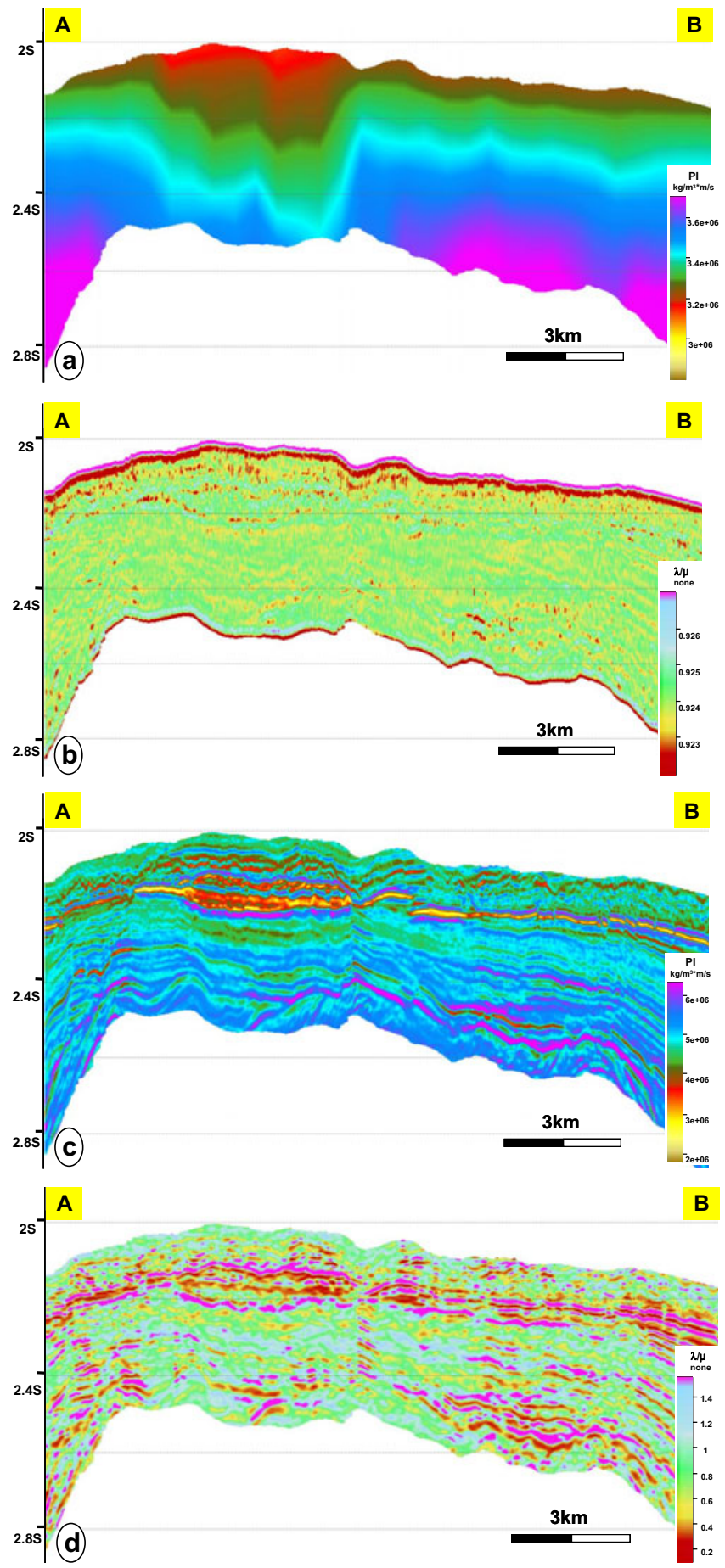

Fig. 9. Comparison between inverted $P I$ and $\lambda / \mu$ results across the same line as in Fig. 2 based on conventional ( $a$ and $b$ ) and proposed (c and d) methods. The resolution of conventional result is much less than that of proposed competitor. Note that the conventional $P I$ result is highly correlated to seismic processing velocity (Fig. 5), and both conventional $P I$ and $\lambda / \mu$ results show very limited distributions of reservoirs, while the new results are more correlative to seismic reflections and are more beneficial for reservoir prediction. 
the conventional pseudo well-logs directly from interval velocities is obviously not applicable. It is not difficult to forecast that the corresponding in vert resolution will not be qualified, and the computed results can hardly end up with good correlations to reservoir parameters. On the contrary, the reconstructed curve by the method we proposed has a higher dominant frequency (same as that of seismic data), as shown The extracted wavelet has produced a higher correlation rate made by correlating synthetic seismograms of reconstructed reflection coefficients and original seismic signals (Fig. 8a,b), which is quite essential to improve the inversion results. A further comparison will be illustrated in Fig. 9.

With these pseudo well-logs, the pre-stack simultaneous AVO/AVA inversion can be conducted to achieve a great number of elastic parameters, such as PI, SI ( $S$-impedance), $V_{p} / V_{s}$, Poisson ratio, $\lambda \rho$, and $\mu \rho$, etc., for reservoir prediction. In particular, both the two sets of pseudo well-logs constructed from the conventional and proposed methods are employed to conduct pre-stack simultaneous AVO/AVA inversion. Figure 9 shows a comparison between the two sets of inverted $P I$ and $\lambda / \mu$ results across the same line based on conventional ( $a$ and $b$ ) and new ( $c$ and $d)$ methods, respectively. Obviously, the resolution of conventional result is much lower than that of proposed competitor, as indicated by these illustrations. In particular, the conventional $P I$ result is highly correlated to seismic processing velocity (Fig. 5), and both conventional $P I$ and $\lambda / \mu$ results have very limited value distributions. On the other hand, the new results by the proposed method are more correlative to seismic reflections and are definitely more beneficial for reservoir prediction.

\section{APPLICATION IN RESERVOIR PREDICTION}

Specifically, as shown in Fig. 10b, the cross-plot of $\lambda / \mu$ and $P I$, that is usually regarded as the best reservoir indicator, is employed for reef and sand reservoir discrimination by separately tracking appropriate data following the yellow and green polygons. The two polygons are similar to that of the nearest drilling place. Thus, the predicted reef (yellow) and sand reservoir (green) can be described, respectively, and elaborately plotted in Fig. 10a. The time thickness maps of predicted reef (Fig. 11b) and sand reservoirs (Fig. 11d) could also be further mapped by summing these qualified samples in a 3D area within two sets of formations, respectively. The two sets of reservoirs have been better delineated by the comparison with their corresponding formation thickness maps (Fig. 11a and c), and they are useful for further exploration of the reservoirs. 


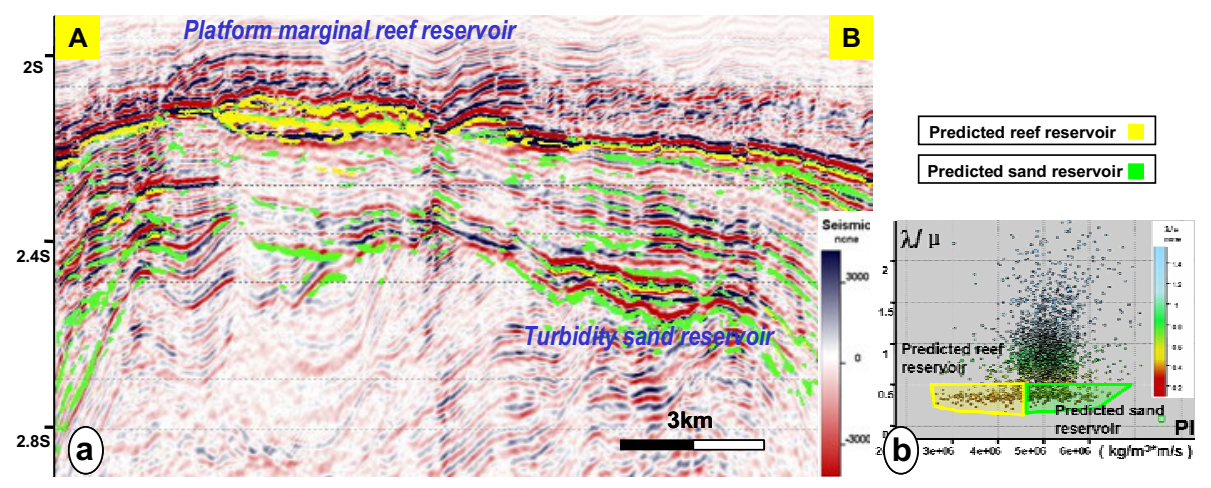

Fig. 10. The cross-plot of inverted $P I$ and $\lambda / \mu$ attributes (b), and the predicted reservoir distributions in the same line as in Fig. 2 (a).
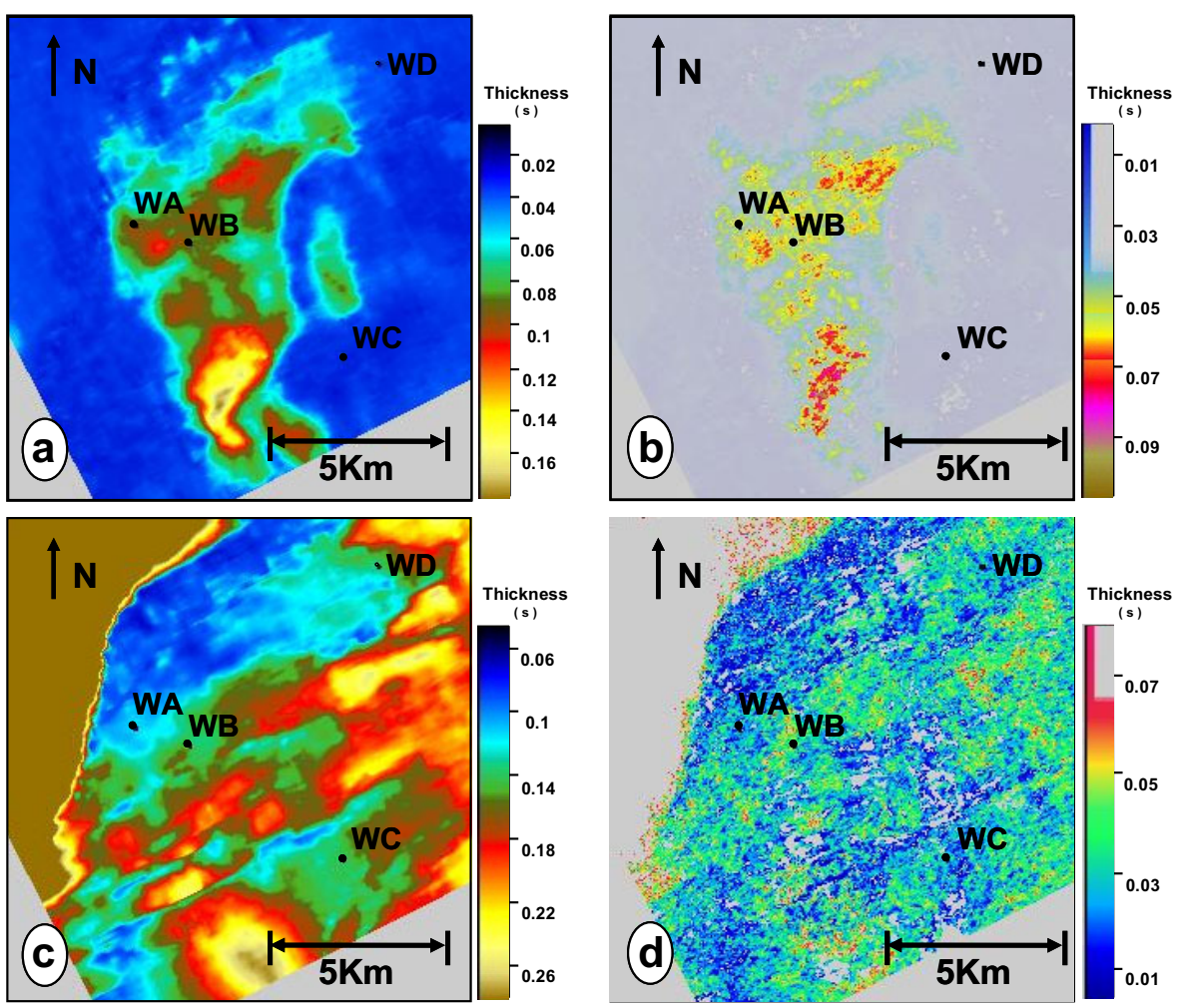

Fig. 11. The time thickness maps of Meishan (T40-T50) (a), and Lingshui (T60T70) fm (c), the predicted reef (b), and turbidity sand (d) reservoir distributions in the two formations, respectively. 


\section{CONCLUSIONS}

It is a common method to construct pseudo well-logs for AVO inversion in unexploited fields that are short of wells, especially in marine cases. But most of conventional methods by directly defining $P$-velocities from seismic processing and computing the $S$-velocity and density using empirical equations are not qualified to scale the extracted source wavelets and build constrained models. They tend to produce very low resolution AVO inversion results. On the contrary, the method we proposed, that reconstructs pseudo well-logs in accordance with reflection amplitudes of computed pure $P$-wave data from CRP gathers (AVO) and drilling information from a close area, is proven to be more beneficial for AVO inversion and more suitable for defining the reservoir prediction parameters. However, it should be noted, in particular, that the CRP data used in this method should have high quality.

Acknowledgments. The authors thank National Basic Research Program of China (Grant No. 2011CB201103), the National Science and Technology Major Project (Grant No. 2011ZX05004003), the Science Foundation of China University of Petroleum-Beijing (KYJJ2012-05-03), and Chinese Scholarship Council for financial support.

\section{References}

Connolly, P. (1999), Elastic impedance, The Leading Edge 18, 4, 438-452, DOI: 10.1190/1.1438307.

Fan, C., Y. Song, Z. Jiang, Y. Zhang, and M. Sun (2013), Reservoir prediction in a well-absent area - a case study in a marine area. In: 75th EAGE Conference and Exhibition incorporating SPE EUROPEC 2013, 10-13 June 2013, London, UK, Extended abstracts, DOI: 10.3997/2214-4609.20130877.

Gidlow, P.M., G.C. Smith, and P.J. Vail (1992), Hydrocarbon detection using fluid factor traces: A case history. In: Joint SEG/EAEG Summer Research Workshop on "How Useful is Amplitude-Versus-Offset (AVO) Analysis?", Expanded abstracts, 78-89.

Hampson, D.P., J.S. Schuelke, and J.A. Quirein (2001), Use of multiattribute transforms to predict log properties from seismic data, Geophysics 66, 1, 220236, DOI: $10.1190 / 1.1444899$.

Hampson, D.P., B.H. Russell, and B. Bankhead (2005), Simultaneous inversion of pre-stack seismic data. In: 75th Annual International Meeting SEG, Expanded abstracts, Society of Exploration Geophysicists, 1633-1637. 
Lamb, W.J., X.H. Zhu, G.A. McMechan, M.L. Greenberg, and J.P. Castagna (1992), Elastic wave propagation in composite media, Geophysics 57, 9, 11551165, DOI: 10.1190/1.1443329.

Sun, Z. (1999), Seismic methods for heavy oil reservoir monitoring and characterization, Ph.D. Thesis, University of Calgary, Calgary, Canada.

Zhang, Y., Z. Sun, C. Fan, and H. Bai (2011a), Data conditioning for pre-stack inversion - a case study from Xingma area, Liaohe Oil field, China. In: 73rd EAGE Conference and Exhibition incorporating SPE EUROPEC 2011, 2326 May 2011, Vienna, Austria, Extended abstracts, DOI: 10.3997/22144609.20149593.

Zhang, Y., Z. Sam, H. Yang, H. Wang, J. Han, H. Gao, C. Luo, and B. Jing (2011b), Pre-stack inversion for caved carbonate reservoir prediction: A case study from Tarim Basin, China, Pet. Sci. 8, 4, 415-421, DOI: 10.1007/s12182011-0159-4.

Zhang, Y., Z. Sun, and C. Fan (2013), An iterative AVO inversion workflow for S-wave improvement. In: 75th EAGE Conference and Exhibition incorporating SPE EUROPEC 2013, 10-13 June 2013, London, UK, Extended abstracts, DOI: 10.3997/2214-4609.20130268.

Received 29 July 2013

Received in revised form 15 April 2014

Accepted 9 June 2014 